\title{
Mandibular morphology, task specialization, and bite mechanics in Pheidole ants
} (Hymenoptera: Formicidae)

Klunk, C.L. ${ }^{1}$; Argenta, M.A. ${ }^{2}$; Casadei-Ferreira, A. ${ }^{3}$; Economo, E.P. ${ }^{3}$; Pie, M.R. ${ }^{1,4}$ ${ }^{1}$ Graduate Program in Ecology and Conservation, Federal University of Paraná, Curitiba-PR,

9 Author for correspondence:

10 Cristian L. Klunk

11 klunkcristian@gmail.com

\section{Abstract}

14 The remarkable ecological and evolutionary success of ants was associated with the evolution

15 of reproductive division of labor, in which sterile workers perform most colony tasks whereas

16 reproductives become specialized in reproduction. In some lineages, the worker force became

17 further subdivided into morphologically distinct subcastes (e.g. minor vs. major workers),

18 allowing for the differential performance of particular roles in the colony. However, the

19 functional and ecological significance of morphological differences between subcastes is not

20 well understood. Here, we applied Finite Element Analysis (FEA) to explore the functional

21 differences between major and minor ant worker mandibles. Analyses were carried out on

22 mandibles of two Pheidole species, a dimorphic ant genus. In particular, we test whether

23 major mandibles evolved to minimize stress when compared to minors using combinations

24 of tooth and masticatory margin bites under strike and pressure conditions. Majors performed

25 better in pressure conditions yet, contrary to our expectations, minors performed better in

26 strike bite scenarios. Moreover, we demonstrate that even small morphological differences

27 in ant mandibles might lead to substantial differences in biomechanical responses to bite 28 loading. These results also underscore the potential of FEA to uncover biomechanical 29 consequences of morphological differences within and between ant worker castes.

30 Key words: Cuticle; Division of labor; Finite Element Analysis; Mandible; Trulleum; 31 Worker polymorphism. 


\section{Introduction}

The evolution of highly complex societies in ants was associated with the evolution

34 of reproductive division of labor, in which sterile workers perform most quotidian colony

35 tasks, whereas reproductives become specialized in colony reproduction (Wilson 1971,

36 Hölldobler \& Wilson 1990). These changes were accompanied by substantial morphological

37 differences among reproductives and workers, with the latter giving up reproduction and

38 dispersal capacities to allow for morphological adaptations that improve the ergonomic

39 efficiency of the colony as a whole. In some ant lineages, the worker force became further

40 subdivided into morphologically distinct subcastes (e.g. minor vs. major workers), and such

41 differences are thought to allow for the differential performance of particular roles in the

42 colony, such as seed milling and colony defense (Wilson 1953; Oster \& Wilson, 1978). In

43 ants, polymorphism evolved in several lineages, and its role to facilitate task specialization

44 is widely recognized (Wilson 1953; Oster \& Wilson, 1978; Wills et al., 2018), and the genetic

45 (Gadagkar 1997; Huang et al., 2013), ecological (Powell \& Franks, 2005; 2006; Powell 2009)

46 and developmental (Wheeler 1991) determinants associated with the occurrence of worker

47 polymorphism have been explored in several ant lineages (Wills et al., 2018).

48 The genus Pheidole shows an interesting pattern among its almost 1,200 known

49 species (Bolton 2020), the development of a dimorphic worker subcaste, represented by

50 major and minor workers, where majors have a disproportionally larger head (Wilson 1953;

51 2003). Pheidole species are distributed worldwide, but most of its diversity and abundance

52 is concentrated in the tropics (Economo et al., 2015a; 2019). Although Pheidole species are

53 typically considered diet generalists (Wilson 2003), some species might show degree of food

54 preferences (Rosumek 2017). Of all their food items, feeding on seeds evolved many times

55 in the genus and has been indicated as an important factor to explain the lineage

56 diversification due to behavioral and morphological adaptations related to seed harvesting

57 and processing (Moreau 2008). Since majors are specialized in tasks as colony defense and

58 food processing (Wilson 1984; 2003), their larger heads could be a consequence of

59 evolutionary pressures towards the specialization to those tasks (Pie \& Traniello, 2007),

60 although evidence gathered so far has been mixed (e.g. Holley et al., 2016).

61 Understanding the main trends in the morphological evolution of Pheidole received

62 considerable attention in the past decade, with different approaches being employed to 
63 understand the evolution of a wide variety of structures, showing contrasting results to the

64 relative contributions of size and shape to the morphological diversity of the genus (Pie \&

65 Traniello, 2007; Pie \& Tschá, 2013; Sarnat et al., 2017; Friedman et al., 2019; 2020), yet

66 little is known about the evolution of mandibular morphology in Pheidole. Ants have a

67 typical pterygote mandible with two articulations with the head (Snodgrass 1935), the dorsal

68 and ventral mandibular joints (Richter et al., 2019). The mandible is the main structure used

69 to interact with the environment (e.g. biting, carrying, excavating, cutting, fighting) (Wheeler

70 1910). Mandibular movement is led by two muscles, the craniomandibularis internus (mci),

71 whose contraction close the mandibles, and the craniomandibularis externus (mce),

72 responsible for the opening process (Snodgrass 1935; Richter et al., 2019). The mci fibers

73 attach to the mandible through a mandibular cuticular projection called apodeme (Paul \&

74 Gronenberg, 1999). The angle of attachment to the apodeme, combined to the sarcomeres

75 length, are directly related to the velocity and force of the mandibular movement (Paul \&

76 Gronenberg, 1999), so that $m c i$ is considered the key to the versatility of ant mandibles

77 (Gronenberg et al., 1997; Paul 2001), being much more developed than the mce (Paul 2001).

78 In Pheidole majors the $m c i$ is remarkably large, where its increase in size in relation to minors

79 happens at the expense of the glandular, digestive, and nervous system in the head (Lillico-

80 Ouachour et al., 2018). Fibers of the mci continue to develop even for days after the adult

81 emergence in both subcastes, and this characteristic correlates to behavioral development in

82 workers (Muscedere et al., 2011).

83 Regardless of the importance of mandibles to many aspects of ant life history, little

84 is known about how the morphological variation between species or worker subcastes relates

85 to bite loads, except for one specialized snap-jaw species (Larabee et al., 2018). Worker

86 polymorphism can lead to behavioral specialization, mainly through variation in mandible

87 morphology (Silva et al., 2016), but biomechanical approaches to directly assess this

88 relationship in ants are scarce (Larabee et al., 2018). To understand how mandible

89 morphology relates to the biomechanical demands of bite, it is important to employ

90 approaches that allow for the direct assessment of bite loading conditions. Finite Element

91 Analysis (FEA) is a numerical method that approximates the mechanical simulation of

92 loading conditions in structures of interest. By applying loads and defining the boundary

93 conditions (movement restrictions) on the structure, FEA estimates the mechanical response, 
94 i.e., how stress flows along the structure according to its shape (Azevedo 2003; Rayfield

95 2007). By employing FEA, one might assess how variation in mandibular morphology

96 among ant species as well as between subcastes translates into the capacity of mandibles to

97 deal with bite loading demands (Larabee et al., 2018), as also explored for the evolution of

98 mandible form in dragonflies (Blanke et al., 2017) and stag beetles (Goyens et al., 2015;

99 2016).

100 To improve our understanding of morphological evolution in Pheidole species, and

101 the role of morphological differentiation to improve task specialization in polymorphic ants,

102 we simulate several bite scenarios in silico by applying FEA (Azevedo 2003; Rayfield 2007)

103 on 3D models of minor and major mandibles of two Pheidole species. We hypothesize that

104 major mandibles are better able to mitigate stress than minors', given their greater robustness.

105 Alternatively, if each worker type has mandibles optimized to perform different tasks, majors

106 and minors could perform better in distinct biting scenarios. Differences between species are

107 expected between the more distinct mandibles of majors, which can suggest changes in the

108 capacity to deal with hard food items, given the specialized roles of those workers (Wilson

109 1984; 2003). Alternatively, differences between species in minor worker mandibles will

110 suggest that even small morphological distinctions can lead to biomechanical idiosyncrasies. 


\section{Methods}

Colonies of Pheidole hetschkoi Emery and P. cf. lucretii were collected in an urban

114 fragment of Atlantic Forest in Curitiba, Paraná, Brazil (25²6'45.9'S 049¹3'55.5”W).

115 Pheidole hetschkoi is commonly found nesting fallen twigs; foraging in the species is based

116 on the recruitment of tens of workers to food sources (rarely majors), and colonies

117 accumulate seeds inside their nests (author's pers. obs.). On the other hand, P. cf. lucretii

118 nests directly on the ground and might recruit up to hundreds of workers to food sources,

119 including dozens of majors, but were never recorded collecting or consuming seeds (author's

120 pers. obs). Morphologically, majors of $P$. hetschkoi are more sturdy, with larger heads

121 (Fig.1b), with more robust mandibles than $P$. cf. lucretii majors, which have also smaller

122 heads and are more slender in general (Fig.1a). Minors differ little between species in terms

123 of mandible shape and general morphology (Fig. 1c and d). Voucher specimens are deposited

124 at the Entomological Collection Padre Jesus Santiago Moure, Department of Zoology,

125 Federal University of Paraná, Brazil.
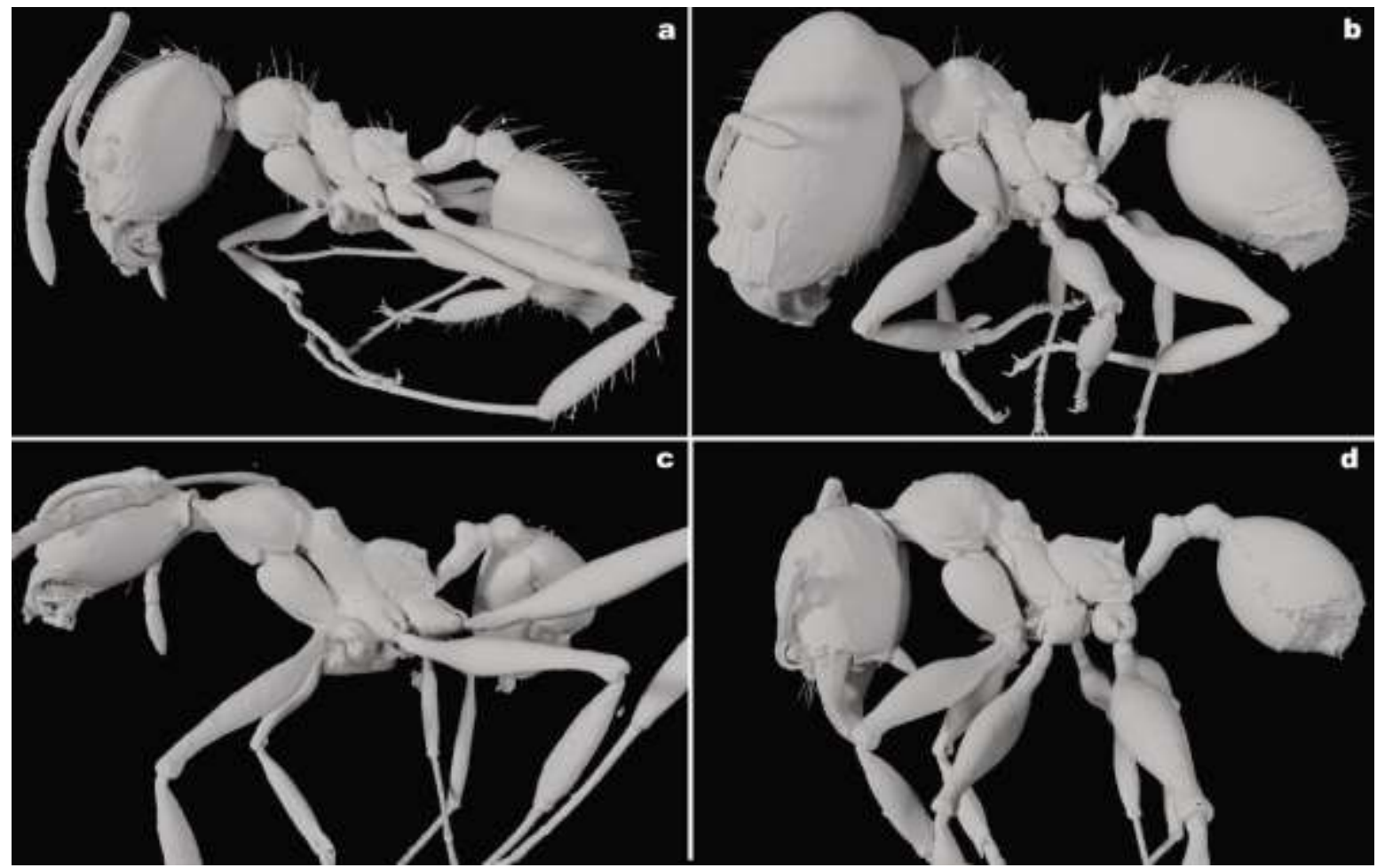

Fig. 1. Pheidole workers whole body 3D models in lateral view. (a) P. cf. lucretii major; (b)

128 P hetschkoi major; (c) P. cf. lucretii minor; (d) P hetschkoi minor. 
132 using the software ZEISS Scout and Scan Control System, in the Biodiversity \& 133 Biocomplexity Unit, Okinawa Institute of Science and Technology Graduate University 134 (OIST), Japan. Exposure time of each specimen varied from one to five seconds, under an 135 "Air" filter and 4x objective. The voltage was set between 30 and 50keV, from 4 to $5 \mathrm{~W}$ of 136 power, under a "normal" field mode and intensity levels of 15,000 and 17,000 across the 137 whole specimen. Scan time varied from 27 to 30 minutes, generating 801 projections from 138 full 360-degree rotations. Model reconstruction was done on XMReconstructor, and 139 mandibles segmentation was carried in ITK-snap 3.8.0 (Yushkevich et al., 2006). For mesh 140 generation and simplification, we used the software MeshLab (Cignoni et al., 2008), and to 141 generate the 3D mandible solid for FEA simulations we used the software Fusion 360 142 (AUTODESK). Mandibles 3D solid models are available on the supplementary material.

\section{FEA simulations}

To quantify the mechanical response of a structure to external loading, FEA requires the discretization of the structure into small parts, resulting in the finite element mesh composed of elements of pre-defined shape and a specific number of points, called nodes, used to solve the equations (Azevedo 2003, Marcé-Nogué et al., 2015). Displacements on nodes are calculated to estimate stress and strain, based on the structure material properties

150 and shape (Rayfield 2007). We used 10-node tetrahedral elements (C3D10) to generate the 151 finite element mesh. The number of elements varied for each model, as well as the size of 152 each element between subcastes, to adapt meshes to each morphology (Table SI1).

We performed linear static simulations of four distinct biting scenarios for each 154 species and subcastes, divided into two categories, namely strike and pressure, which reflect 155 different aspects of mandible movement in terms of force and velocity. In strike scenarios, a 156 condition associated with faster movements, we define the mandible articulations with the 157 head (dorsal - $d m a$ and ventral - vma) as the constrained regions, applying static load on the 158 apical tooth or the masticatory margin (Figure 2a, b). In pressure scenarios, associated with 159 slower mandible movements but powerful bites, in addition to the mandibular joints, we also 
160 constrained the apical tooth or the masticatory margin and applied the load to the region of

$161 m c i$ insertion, following the direction of contraction (Figure 2a, c) to simulate the use of 162 mandibles for food compression. We constrained nodal displacement in $\mathrm{x}, \mathrm{y}$, and $\mathrm{z}$ directions

163 and apply a $1 \mathrm{~N}$ load uniformly distributed among nodes in all simulations. We modeled the

164 mandible cuticle as an isotropic and linearly elastic material, setting Young's modulus as

$1652.75 \mathrm{GPa}$ and the Poisson's ratio as 0.3 , based on measures from the cuticle of ant mandibles

166 available in the literature (Brito et al., 2017). The only source of variation for each biting

167 simulation between species and workers was the morphology of the mandibles. We present

168 FEA stress results based on Tresca failure criterion, more suitable for brittle fracture, which

169 determine an equivalent stress value under which the material will possibly fail when

170 subjected to combined load (Özkaya et al., 2017). We used Abaqus 6 (Dassault Systèmes) to

171 run the FEA simulations. 


\section{a}

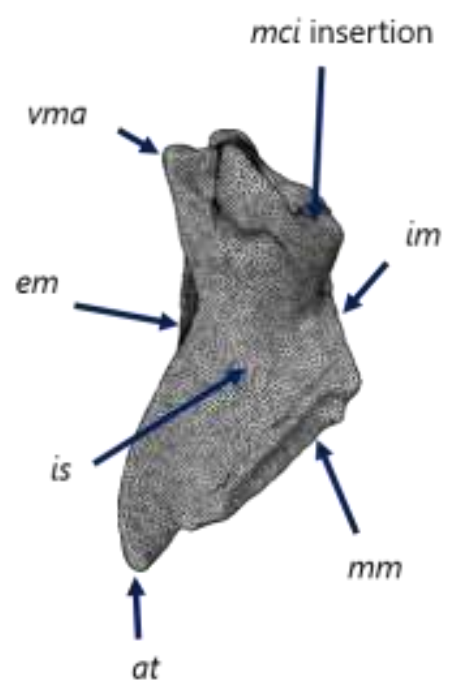

(b)
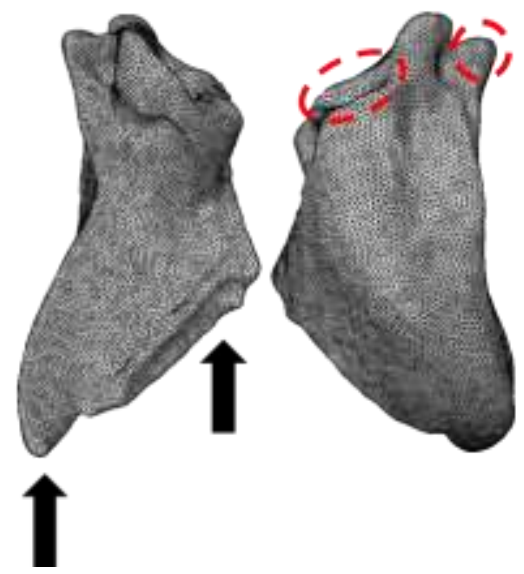

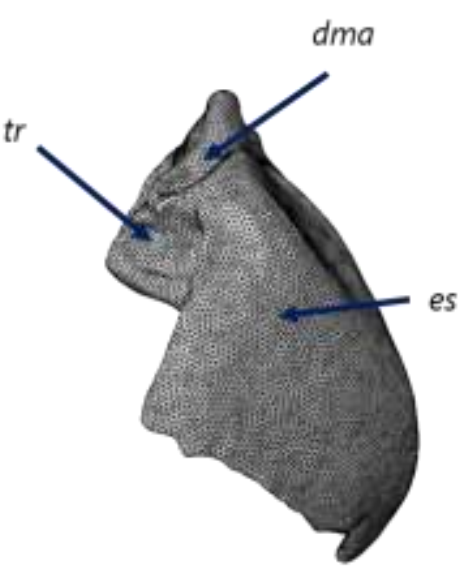

C
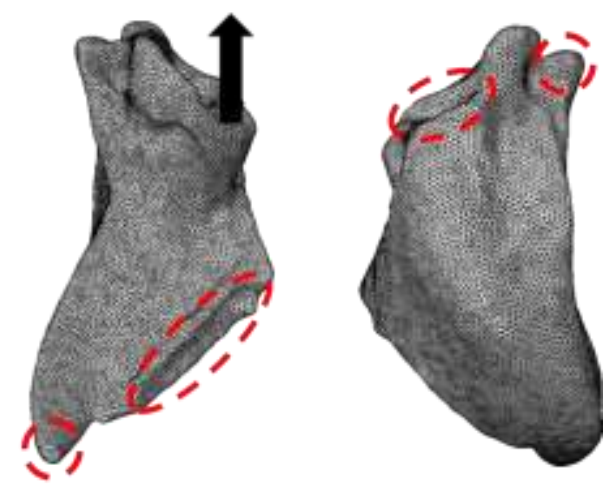

173 Fig. 2: Main mandibular regions considered to discuss stress dissipation patterns (a); Loaded

174 and constrained regions in strike (b) and pressure (b) biting simulations. In b and c, arrows

175 indicate the direction and region of load, and dashed lines enclose the constrained regions for

176 each simulation. at: apical tooth; dma: dorsal mandibular articulation; em: external margin;

177 es: external surface; im: internal margin; is: internal surface; $m c i$ : muscle craniomandibularis

178 internus; $m m$ : masticatory margin; $t r$ : trulleum; $v m a$ : ventral mandibular articulation. 
179

180

181

182

183

184

185

186

\section{Results}

FEA simulations

Stress distribution results are shown in Figure 3. Given that the volume of each model varies, and that we use idealized loads and material properties, we chose not to interpret absolute stress values. Rather, we will focus on qualitative differences among simulations by rescaling the stress ranges based on a reference model to facilitate comparisons between species, subcastes, and biting scenarios. Therefore, relative differences in stress distribution between simulations indicate mandibular biomechanical distinctions to assimilate loading conditions.

\section{Major worker mandibles}

When displacement restrictions were applied on the mandibular joints, those regions expectedly showed high-stress levels, but stresses had to spread to other regions to be effectively absorbed. Starting from the dorsal mandibular articulation ( $d m a)$, stresses dissipate mainly along the mandible external surface and trulleum (Fig. 3c, d, g, h, k, 1, o, p). Indeed, the trulleum was important to concentrate stresses coming from the dma in all simulations. Stresses from the ventral mandibular articulation ( $v m a$ ) spread mainly along the external margin and through its surroundings along internal and external surfaces (Fig. 3c, d, $\mathrm{g}, \mathrm{h}, \mathrm{k}, \mathrm{l}, \mathrm{o}, \mathrm{p})$. Contrasting different biting scenarios, higher stresses are found when only the apical tooth is employed (Fig. 3c, d, k, l). This result indicates that ants face marked mechanical restrictions when they only use the apical tooth. Pressure scenarios generated higher stresses around the basal region of the internal surface (Fig. 3k, 1, o, p), whereas strike scenarios concentrated more stress near the masticatory margin, an expected consequence of load application (Fig. 3c, d, g, h). However, the key aspect relating different biting scenarios are the higher stress levels in $d m a$ and $v m a$ in the strike (Fig. $3 \mathrm{c}, \mathrm{d}, \mathrm{g}, \mathrm{h}$ ) versus pressure simulations (Fig. 3k, 1, o, p), which indicates that strike causes higher mechanical demands in the mandibular joints than pressure.

The main aspect that influences stress dissipation differences between species is the mandible internal surface concavity. Pheidole hetschkoi has a deeper concavity near the masticatory margin, which acts as an important stress concentrator, mainly in strike scenarios on the masticatory margin (Fig. $2 \mathrm{~g}$ ). While $P$. cf. lucretii also shows stress concentration at 
210 the same region in this biting scenario, those stresses spread more extensively along the

211 internal surface (Fig. 3h), which suggests that its concavity is shallow and does not act as a

212 stress concentrator. The external surface curvature also differs between species, but there are

213 no substantial differences in terms of stress dissipation pattern (Fig. 3c, d, g, h, k, 1, o, p). The

214 dissipation through the external surface is more restricted to the articulations surroundings,

215 given the robustness of the mandibular base, which could explain why there is not a

216 conspicuous effect of the external surface curvature in the stress dissipation pattern between

217 species. Stresses were proportionally higher in the $P$. cf. lucretii mandible, through most

218 mandibular regions and all biting scenarios, but the differences are more striking in pressure

219 scenarios (Fig. 31, p).

\section{Minor worker mandibles}

There is a distinguished stress concentration around the more constricted region of the mandibular internal surface, a trend that occurs mainly in strike simulations, especially when the load was applied on the masticatory margin (Fig. 3e, f). This constriction acts as a stress concentrator in minors due to their slender mandibles in comparison to majors. When the results of different species are compared, $P$. cf. lucretii simulations show proportionately higher stresses than $P$. hetschkoi in general (Fig. 3b, f, j, n), contrary to the expectation that minors mandibles would not differ in mechanical performance. The overall lower stress levels found in masticatory margin strike simulations of $P$. hetschkoi minors seems to reflect the presence of well-developed teeth along its masticatory margin. It is noticeable that the masticatory margin teeth absorb great levels of stress (Fig. 3e), so that their absence leads to higher stress levels along the mandible surfaces in strike simulations of $P$. cf. lucretii minor, as well as in majors of both species. The higher stresses along the internal surface in $P$. cf. lucretii minor mandible, compared to P. hetschkoi minor mandible, draw attention to the mechanical limitations associated with worn mandibles, as is the case of the P. cf. lucretii minor mandible modeled, which can lead to behavioral switches in task performance along the worker lifetime. Regarding the biting scenarios, pressure in minors result in higher stresses on internal and external surfaces of both species when compared to majors (Fig. 3i, $\mathrm{j}, \mathrm{m}, \mathrm{n})$. As occurred in pressure scenarios for majors, stresses along the internal surface concentrate near the mandible base, where the load was applied. However, in minors, the 
bioRxiv preprint doi: https://doi.org/10.1101/2020.11.16.385393; this version posted November 17,2020 . The copyright holder for this preprint (which was not certified by peer review) is the author/funder, who has granted bioRxiv a license to display the preprint in perpetuity. It is made available under aCC-BY-NC-ND 4.0 International license.

241 mandible base is slender, which can explain why the mandibular surfaces in minors are 242 proportionally more stressed in pressure than in strike simulations. 


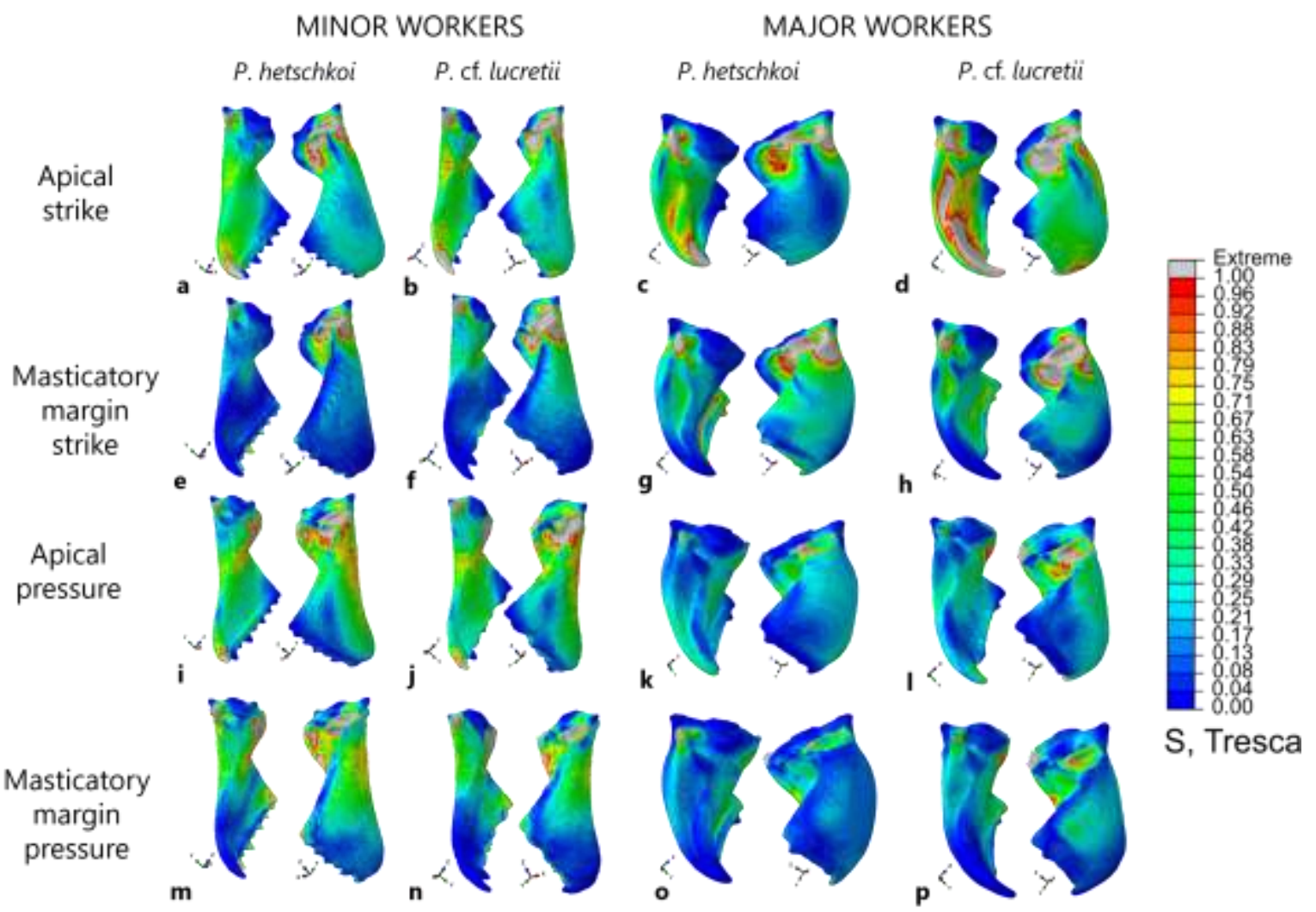

244 Fig. 3: Tresca stress results (rescaled to range between 0-1) for the four biting scenarios

245 (rows), from minors and majors of both Pheidole species (columns). Each letter depicts a

246 distinct simulation. Color represents a proportional value of stress in relation to the maximum

247 value considered for each simulation, indicated as 1.00, and grey represents extremes values

248 above the maximum considered. 


\section{Discussion}

The use of biomechanical simulations to explore the relationships between form and

251 function in insects is a recent endeavor. To date, FEA simulations have been used to

252 understand biomechanical consequences of male stag beetle mandibles during agonistic

253 interactions (Goyens et al., 2014; 2015; 2016), as well as the morphological evolution of

254 Anisoptera (Odonata) mandibles (Blanke et al., 2017). More recently, it was demonstrated

255 how mandibles of the trap-jaw ant Mystrium camillae are morphologically adapted to deal

256 with the loadings arising from the power amplification mechanism of its closing movement

257 (Larabee et al., 2018). Here we apply FEA in mandibles of Pheidole workers to simulate

258 different biting scenarios and investigate how morphological differences in mandible

259 morphology reflects their responses to those bite loading demands.

260 Our results demonstrate how mandible morphology of dimorphic workers can be

261 optimized for particular tasks and draws attention to the role of specific mandibular regions

262 or structures to deal with the stresses generated by their bite. Ant workers have a typical

263 pterygote triangular mandible (Snodgrass, 1935), which can be divided into two components,

264 a basal thick stem, and a distal blade (Richter et al., 2020). Our results indicate that this

265 distribution of cuticular material in the mandible may conform to the high loading demands

266 experienced by the mandibular articulations with the head. Most of the stresses generated on

267 the apical tooth dissipate along the external margin towards the mandibular base, in both

268 species and subcastes, avoiding the spread of considerable stresses through the more delicate

269 mandibular surfaces. In masticatory margin stress simulations, the presence of well-

270 developed teeth results in stresses being concentrated on the teeth instead of spreading

271 through the internal surface. Majors of Pheidole, in which the masticatory margin is

272 toothless, show a higher degree of concavity on their internal surfaces, especially in $P$.

273 hetschkoi, which helps to concentrate stresses near the more robust masticatory margin

274 instead of spreading through the internal surface. Although alleviating the level of stress in

275 the mandibular articulations, such stress concentration can be harmful in cases in which the

276 structure is submitted to cycles of loading, leading to structural fail due to material fatigue

277 (Dirks et al., 2013).

278 An important aspect of Pheidole mandibular morphology with respect to bite

279 mechanics is the role of the trulleum on stress concentration. The trulleum is a concavity near 
the dma present only in some myrmicine ants (Richter et al., 2019). The function of the trulleum was hitherto unknown. Here we demonstrate for the first time the importance of the trulleum to concentrate stresses coming from the $d m a$, avoiding the spread of stresses through the more delicate mandibular surfaces. This is an interesting discovery, given that the $d m a$ seems to concentrate higher stresses in general than the vma. Given the trulleum clear functional role here outlined, it would be interesting to investigate the biomechanical responses of mandibles that lack the development of the trulleum, to understand how stresses dissipate from dma without this important stress concentrator, especially in ant species with similar loading demands as Pheidole mandibles.

Our results also underscore how the more robust major mandibles are better suited to deal with pressure biting than minors slender mandibles, which show higher performance in strike scenarios. As expected, these results agree with the specialized roles played by major workers in the colony. The behavioral repertoire of major workers is particularly limited, 293 being frequently restricted to defense and/or food processing (Wilson 1984; 2003). Indeed, 294 when minors are experimentally removed from the colony, major workers take over many of their tasks, although with decreased efficiency (Wilson 1984; Mertl \& Traniello, 2009). Major mandibles meet the demands to deal with the processing of hard food items through pressure, with their toothless masticatory margin spreading bite forces evenly around the food item. Seed consumption is considered an important aspect in the evolution of several myrmicine genera, such as Pheidole, Pogonomyrmex, and Solenopsis (Ferster et al., 2006; Moreau 2008). However, the influence of granivory on morphological evolution, especially

301 regarding the dimorphism in the Pheidole worker caste, is still poorly known (Holley et al.,

302 2016). Here, we demonstrate for the first time how ant mandible morphology can be tuned 303 to deal with the mechanical demands of processing hard food items, such as seeds and 304 arthropod cuticles, through major mandibles better performance in pressure biting conditions. 305 Also, mandibles of $P$. hetschkoi majors show an even better performance in pressure bite 306 than $P$. cf. lucretii, suggesting that majors of $P$. hetschkoi can deal better with harder food 307 items than $P$. cf. lucretii. These results may lead to the possibility of food partitioning among 308 Pheidole coexisting species, and agrees with the habit of seed consumption by P. hetschkoi, 309 which demands higher bite forces and consequently leads to higher stress levels on the 310 mandibles. 
Although minor workers of Pheidole typically show well-developed teeth in their

312 masticatory margin, the particular specimen of $P$. cf. lucretii included in our study showed

313 high levels of teeth wear, allowing us to assess the consequences of teeth wear on bite

314 loadings. Teeth concentrate the forces generated by the masticatory muscles on smaller areas,

315 improving the initiation of the fracture (Clissold 2007). The importance of teeth to task

316 efficiency was demonstrated for leaf-cutting ants, where workers specialized to cut leaves

317 switch to carrying them once their teeth are worn to a certain degree, reducing their cutting

318 efficiency (Schofield et al., 2011). In Pheidole, minors perform a wide range of tasks in the

319 colony (Wilson 1984; 2003), but information on the role of teeth wear in minor task switching

320 is scarce. Here we demonstrate the possible mechanical consequences of teeth wear in ant

321 mandibles, comparing the relative amount of stress generated during masticatory margin

322 strike simulations in $P$. hetschkoi and $P$. cf. lucretii minors. Our results indicated that $P$. cf.

323 lucretii has relatively higher stresses than $P$. hetschkoi, mainly along its mandible internal

324 surface, which drives to higher stresses at the mandibular articulations with the head. Further

325 studies in task allocation and mandible morphology in dimorphic ants colonies can address

326 if teeth wear generates task switch, and biomechanical studies can reveal how teeth wear

327 reduces task efficiency (Schofield et al., 2011).

328 The morphological evolution of Pheidole might be strongly driven by differences in

329 size (Pie \& Traniello, 2007), which tends to evolve at higher rates than shape (Pie \& Tschá,

330 2013; Economo et al., 2015a; Friedman et al., 2019). More recently, studies applying

331 geometric morphometric approaches validated the prominence of size to explain the

332 morphological disparity in the genus but also pointed to different evolutionary rates and

333 levels of integration between head and mesosoma shape and size (Friedman et al., 2019;

334 2020). Pheidole morphological diversification seems to be very constrained (Pie \& Traniello,

335 2007), in contrast to their ecological disparity (Economo et al., 2015a; 2015b), as reflected

336 in the widespread distribution of genus throughout most of the terrestrial ecosystems

337 (Economo et al., 2019). Field observations demonstrate that, despite the relative

338 morphological resemblance in Pheidole species, they can show considerable ecological and

339 behavioral diversity (Mertl et al., 2010; Tschá \& Pie, 2019). We demonstrate that even small

340 morphological differences in mandibles shape between species can lead to biomechanical

341 specialization, mainly in terms of the food processing capacity of majors. This biomechanical 
342 specialization can expand the diet range of species and lead to food partitioning (Blüthgen et

343 al., 2003; Rosumek 2017), decreasing the degree of competition and allowing for species

344 coexistence (Blüthgen \& Feeldar, 2010).

345 Our results provide a biomechanical basis to understand how mandible 346 morphological evolution can improve task specialization in polymorphic ants. Morphological 347 polymorphism in the worker caste can expand the range of prey items that a species is able 348 to handle, as demonstrated for some species of the army ant genus Eciton (Powell \& Franks, $3492005 ;$ 2006). In the highly polymorphic genus Cephalotes, which is Pheidole's sister lineage, 350 some workers are specialized to plug the nest entrances with their heads to protect the colony 351 against invasion (Powell 2008). In some fire ants such as Solenopsis geminate (Fabricius), 352 the degree of worker polymorphism is associated to higher levels of division of labor, with 353 major workers being specialized to seed milling (Wilson 1978; Ferster et al., 2006). Division 354 of labor in leaf-cutting ants is associated with morphological distinctions among worker 355 mandibles, as demonstrated for the polymorphic genus Atta (Silva et al., 2016). Therefore, 356 the role of worker polymorphism for division of labor in ants is well established (Wills et al., 357 2018), but by applying biomechanical approaches we can advance our understanding about 358 the functional role of morphological disparity, as we demonstrated here for Pheidole workers. 359 In this sense, ant polymorphic lineages are ideal models to investigate form-function 360 relationships, and the morphological differentiation of mandibles should be studied in detail, 361 given the importance of this structure to worker interactions with the environment.

\section{Acknowledgments}

364 The authors acknowledge Dr. Emily Rayfield, Dr. Mauricio Moura and Dr. Roberto Keller 365 for valuable comments on our manuscript. Thanks to the CAPES Foundation for the support 366 provided to CLK (doctorate scholarship - 001) and ACF (PDSE grant 88881.189085/2018367 01). E.P.E. was supported by subsidy funding to OIST. The authors thank the OIST Imaging 368 section for access to the CT scanner.

\section{References}

370 Azevedo AF. 2003 Método dos Elementos Finitos. Porto: Faculdade de Engenharia da 371 Universidade do Porto. 
372 Blanke A, Schmitz H, Patera A, Dutel H, Fagan MJ. 2017 Form-function relationships in

373 dragonfly mandibles under an evolutionary perspective. J. R. Soc. Interface 14, 20161038.

374 (doi:10.1098/rsif.2016.1038)

375 Blüthgen N, Feldhaar H. 2010 Food and shelter: how resources influence ant ecology. In Ant 376 Ecology, pp. 115-136. New York: Oxford University Press.

377 Blüthgen N, Gebauer G, Fiedler K. 2003 Disentangling a rainforest food web using stable 378 isotopes: dietary diversity in a species-rich ant community. Oecologia 137, 426-435. 379 (doi:10.1007/s00442-003-1347-8)

380 Bolton B. An online catalog of the ants of the world. 2020. antcat.org (last access in 381 03/SEP/2020).

382 Brito TO, Elzubair A, Araújo LS, Camargo SA de S, Souza JLP, Almeida LH. 2017 383 Characterization of the Mandible Atta Laevigata and the Bioinspiration for the Development 384 of a Biomimetic Surgical Clamp. Mat. Res. 20, 1525-1533. (doi:10.1590/1980-5373-mr385 2016-1137)

386 Cignoni P, Callieri M, Corsini M, Dellepiane M, Ganovelli F, Ranzuglia G. 2008 Meshlab: 387 an open-source mesh processing tool. In Eurographics Italian chapter conference, pp. 129388136.

389 Clissold FJ. 2007 The biomechanics of chewing and plant fracture: mechanisms and 390 implications. Adv In Insect Phys 34, 317-372.

391 Dirks J-H, Parle E, Taylor D. 2013 Fatigue of insect cuticle. Journal of Experimental Biology 392 216, 1924-1927. (doi:10.1242/jeb.083824)

393 Economo EP, Huang J-P, Fischer G, Sarnat EM, Narula N, Janda M, Guénard B, Longino 394 JT, Knowles LL. 2019 Evolution of the latitudinal diversity gradient in the hyperdiverse ant 395 genus Pheidole. Global Ecol Biogeogr 28, 456-470. (doi:10.1111/geb.12867)

396 Economo EP, Klimov P, Sarnat EM, Guénard B, Weiser MD, Lecroq B, Knowles LL. 2015a 397 Global phylogenetic structure of the hyperdiverse ant genus Pheidole reveals the repeated 398 evolution of macroecological patterns. Proc. R. Soc. B 282, 20141416. 399 (doi:10.1098/rspb.2014.1416) 
400 Economo EP et al. 2015b Breaking out of biogeographical modules: range expansion and

401 taxon cycles in the hyperdiverse ant genus Pheidole. J. Biogeogr. 42, 2289-2301.

402 (doi:10.1111/jbi.12592)

403 Evans AR, Sanson GD. 2005 Biomechanical properties of insects in relation to insectivory: 404 cuticle thickness as an indicator of insect 'hardness' and 'intractability'. Aust. J. Zool. 53, 9. 405 (doi:10.1071/ZO04018)

406 Ferster B, Pie MR, Traniello JFA. 2006 Morphometric variation in North American 407 Pogonomyrmex and Solenopsis ants: caste evolution through ecological release or dietary 408 change? Ethology Ecology \& Evolution 18, 19-32. (doi:10.1080/08927014.2006.9522723)

409 Friedman NR, Lecroq Bennet B, Fischer G, Sarnat EM, Huang J, Knowles LLK, Economo 410 EP. 2020 Macroevolutionary integration of phenotypes within and across ant worker castes.

411 Ecol Evol, ece3.6623. (doi:10.1002/ece3.6623)

412 Friedman NR, Remeš V, Economo EP. 2019 A morphological integration perspective on the 413 evolution of dimorphism among sexes and social insect castes. Integr Comp Biol 59, 410414419.

415 Gadagkar R. 1997 The evolution of caste polymorphism in social insects: Genetic release 416 followed by diversifying evolution. J. Genet. 76, 167-179. (doi:10.1007/BF02932215)

417 Goyens J, Dirckx J, Aerts P. 2015 Built to fight: variable loading conditions and stress 418 distribution in stag beetle jaws. Bioinspir. Biomim. 10, 046006. (doi:10.1088/1748$4193190 / 10 / 4 / 046006)$

420 Goyens J, Dirckx J, Aerts P. 2016 Jaw morphology and fighting forces in stag beetles. J Exp 421 Biol 219, 2955-2961. (doi:10.1242/jeb.141614)

422 Goyens J, Soons J, Aerts P, Dirckx J. 2014 Finite-element modelling reveals force 423 modulation of jaw adductors in stag beetles. J. R. Soc. Interface 11, 20140908. 424 (doi:10.1098/rsif.2014.0908)

425 Gronenberg W, Paul J, Just S, Hölldobler B. 1997 Mandible muscle fibers in ants: fast or 426 powerful? Cell and Tissue Research 289, 347-361. (doi:10.1007/s004410050882) 
427 Hao W, Yao G, Zhang X, Zhang D. 2018 Kinematics and Mechanics analysis of trap-jaw ant

428 Odontomachus monticola. J. Phys.: Conf. Ser. 986, 012029. (doi:10.1088/1742-

$4296596 / 986 / 1 / 012029)$

430 Hölldobler B, Wilson EO. 1990 The ants. Cambridge, Mass: Belknap Press of Harvard 431 University Press.

432 Holley J-AC, Moreau CS, Laird JG, Suarez AV. 2016 Subcaste-specific evolution of head 433 size in the ant genus Pheidole. Biol. J. Linn. Soc. 118, 472-485. (doi:10.1111/bij.12769)

434 Huang MH, Wheeler DE, Fjerdingstad EJ. 2013 Mating system evolution and worker caste 435 diversity in Pheidole ants. Mol Ecol 22, 1998-2010. (doi:10.1111/mec.12218)

436 Larabee FJ, Smith AA, Suarez AV. 2018 Snap-jaw morphology is specialized for high-speed

437 power amplification in the Dracula ant, Mystrium camillae. R. Soc. open sci. 5, 181447. 438 (doi:10.1098/rsos.181447)

439 Lillico-Ouachour A, Metscher B, Kaji T, Abouheif E. 2018 Internal head morphology of 440 minor workers and soldiers in the hyperdiverse ant genus Pheidole. Can. J. Zool. 96, 383441 392. (doi:10.1139/cjz-2017-0209)

442 Marcé-Nogué J, Fortuny J, Gil L, Sánchez M. 2015 Improving mesh generation in finite 443 element analysis for functional morphology approaches. Spanish J. Palaeontol. 30, 117-132. 444 (doi:10.7203/sjp.30.1.17227)

445 Mertl AL, Sorenson MD, Traniello JFA. 2010 Community-level interactions and functional 446 ecology of major workers in the hyperdiverse ground-foraging Pheidole (Hymenoptera, 447 Formicidae) of Amazonian Ecuador. Insect. Soc. 57, 441-452. (doi:10.1007/s00040-010$448 \quad 0102-5)$

449 Mertl AL, Traniello JFA. 2009 Behavioral evolution in the major worker subcaste of twig450 nesting Pheidole (Hymenoptera: Formicidae): does morphological specialization influence 451 task plasticity? Behav Ecol Sociobiol 63, 1411-1426. (doi:10.1007/s00265-009-0797-3)

452 Moreau CS. 2008 Unraveling the evolutionary history of the hyperdiverse ant genus Pheidole 453 (Hymenoptera: Formicidae). Molecular Phylogenetics and Evolution 48, 224-239. 454 (doi:10.1016/j.ympev.2008.02.020) 
455 Muscedere ML, Traniello JFA, Gronenberg W. 2011 Coming of age in an ant colony: 456 cephalic muscle maturation accompanies behavioral development in Pheidole dentata. 457 Naturwissenschaften 98, 783-793. (doi:10.1007/s00114-011-0828-6)

458 Neville AC. 1975 General Structure of Integument. In Biology of the Arthropod Cuticle, pp. 459 7-70. Berlin: Springer - Verlag.

460 Oliver WC, Pharr GM. 1992 An improved technique for determining hardness and elastic 461 modulus using load and displacement sensing indentation experiments. J. Mater. Res. 7, 462 1564-1583. (doi:10.1557/JMR.1992.1564)

463 Oster GF, Wilson EO 1978 Caste and Ecology in the Social Insects. New Jersey: Princeton 464 University Press.

465 Özkaya N, Leger D, Goldsheyder D, Nordin M. 2017 Multiaxial Deformations and Stress 466 Analyses. In Fundamentals of Biomechanics: Equilibrium, Motion, and Deformation, pp. 467 317-360. New York: Springer.

468 Paul J. 2001 Mandible movements in ants. Comparative Biochemistry and Physiology Part 469 A: Molecular \& Integrative Physiology 131, 7-20. (doi:10.1016/S1095-6433(01)00458-5)

470 Paul J, Gronenberg W. 1999 Optimizing force and velocity: mandible muscle fibre 471 attachments in ants. Journal of Experimental Biology 202, 797-808.

472 Pie MR, Traniello JFA. 2007 Morphological evolution in a hyperdiverse clade: the ant genus 473 Pheidole. J Zoology 271, 99-109. (doi:10.1111/j.1469-7998.2006.00239.x)

474 Pie MR, Tschá MK. 2013 Size and shape in the evolution of ant worker morphology. PeerJ 475 1, e205. (doi:10.7717/peerj.205)

476 Powell S, Franks NR. 2005 Caste evolution and ecology: a special worker for novel prey. 477 Proc. R. Soc. B 272, 2173-2180. (doi:10.1098/rspb.2005.3196)

478 Powell S. 2008 Ecological specialization and the evolution of a specialized caste in 479 Cephalotes ants. Functional Ecology 22, 902-911. (doi:10.1111/j.1365-2435.2008.01436.x)

480 Powell S, Franks NR. 2006 Ecology and the evolution of worker morphological diversity: a 481 comparative analysis with Eciton army ants. Funct Ecology 20, 1105-1114. 482 (doi:10.1111/j.1365-2435.2006.01184.x) 
483 Powell S. 2009 How ecology shapes caste evolution: linking resource use, morphology, 484 performance and fitness in a superorganism. Journal of Evolutionary Biology 22, 1004-1013. 485 (doi:10.1111/j.1420-9101.2009.01710.x)

486 Rajabi H, Ghoroubi N, Darvizeh A, Dirks J-H, Appel E, Gorb SN. 2015 A comparative study 487 of the effects of vein-joints on the mechanical behaviour of insect wings: I. Single joints. 488 Bioinspir. Biomim. 10, 056003. (doi:10.1088/1748-3190/10/5/056003)

489 Rajabi H, Ghoroubi N, Darvizeh A, Appel E, Gorb SN. 2016 Effects of multiple vein 490 microjoints on the mechanical behaviour of dragonfly wings: numerical modelling. $R$. Soc. 491 open sci. 3, 150610. (doi:10.1098/rsos.150610)

492 Rayfield EJ. 2007 Finite Element Analysis and Understanding the Biomechanics and 493 Evolution of Living and Fossil Organisms. Annu. Rev. Earth Planet. Sci. 35, 541-576. 494 (doi:10.1146/annurev.earth.35.031306.140104)

495 Richter A, Hita Garcia F, Keller RA, Billen J, Economo EP, Beutel RG. 2020 Comparative 496 analysis of worker head anatomy of Formica and Brachyponera (Hymenoptera: Formicidae). 497 Arthropod Systematics \& Phylogeny 78, 133-170. (doi:10.26049/ASP78-1-2020-06)

498 Richter A, Keller RA, Rosumek FB, Economo EP, Hita Garcia F, Beutel RG. 2019 The 499 cephalic anatomy of workers of the ant species Wasmannia affinis (Formicidae, 500 Hymenoptera, Insecta) and its evolutionary implications. Arthropod Structure \& 501 Development 49, 26-49. (doi:10.1016/j.asd.2019.02.002)

502 Rosumek FB. 2017 Natural History of Ants: What We (do not) Know About Trophic and 503 Temporal Niches of Neotropical Species. Sociobiology 64, 244. 504 (doi:10.13102/sociobiology.v64i3.1623)

505 Sarnat EM, Friedman NR, Fischer G, Lecroq-Bennet B, Economo EP. 2017 Rise of the spiny 506 ants: diversification, ecology and function of extreme traits in the hyperdiverse genus 507 Pheidole (Hymenoptera: Formicidae). Biological Journal of the Linnean Society 122, 514508 538. (doi:10.1093/biolinnean/blx081)

509 Schofield RMS, Emmett KD, Niedbala JC, Nesson MH. 2011 Leaf-cutter ants with worn 510 mandibles cut half as fast, spend twice the energy, and tend to carry instead of cut. Behav 511 Ecol Sociobiol 65, 969-982. (doi:10.1007/s00265-010-1098-6) 
512 Silva LC, Camargo RS, Lopes JFS, Forti LC. 2016 Mandibles of Leaf-Cutting Ants:

513 Morphology Related to Food Preference. Sociobiology 63, 881.

514 (doi:10.13102/sociobiology.v63i3.1014)

515 Sirohi J, Chopra I. 2000 Fundamental Understanding of Piezoelectric Strain Sensors. Journal

516 of Intelligent Material Systems and Structures 11, 246-257. (doi:10.1106/8BFB-GC8P$517 \quad \mathrm{XQ} 47-\mathrm{YCQ} 0)$

518 Snodgrass RE, 1935 Principles of Insect Morphology. New York: Cornell University Press.

519 Sun J, Tong J, Ma Y. 2008 Nanomechanical Behaviours of Cuticle of Three Kinds of Beetle.

520 Journal of Bionic Engineering 5, 152-157. (doi:10.1016/S1672-6529(08)60087-6)

521 Tschá MK, Pie MR. 2019 Correlates of ecological dominance within Pheidole ants

522 (Hymenoptera: Formicidae): Correlates of ecological dominance in ants. Ecol Entomol 44, 523 163-171. (doi:10.1111/een.12685)

524 Vincent JFV, Wegst UGK. 2004 Design and mechanical properties of insect cuticle.

525 Arthropod Structure \& Development 33, 187-199. (doi:10.1016/j.asd.2004.05.006)

526 Yushkevich PA, Piven J, Hazlett HC, Smith RG, Ho S, Gee JC, Gerig G. 2006 User-guided 527 3D active contour segmentation of anatomical structures: Significantly improved efficiency 528 and reliability. NeuroImage 31, 1116-1128. (doi:10.1016/j.neuroimage.2006.01.015)

529 Wheeler DE. 1991 The Developmental Basis of Worker Caste Polymorphism in Ants. The 530 American Naturalist 138, 1218-1238. (doi:10.1086/285279)

531 Wheeler WM. 1910. Ants: their structure, development and behavior. New York: Columbia 532 University Press.

533 Wills BD, Powell S, Rivera MD, Suarez AV. 2018 Correlates and Consequences of Worker 534 Polymorphism in Ants. Annu. Rev. Entomol. 63, 575-598. (doi:10.1146/annurev-ento535 020117-043357)

536 Wilson EO. 1978 Division of labor in fire ants based on physical castes (Hymenoptera: 537 Formicidae: Solenopsis). Journal of the Kansas Entomological Society 51, 615-636.

538 Wilson EO. 2003 Pheidole in the New World: a dominant, hyperdiverse ant genus. 539 Cambridge, Mass: Harvard University Press. 
540 Wilson EO. 1971 The insect societies. Cambridge, EUA: Harvard University Press.

541 Wilson EO. 1953 The Origin and Evolution of Polymorphism in Ants. The Quarterly Review

542 of Biology 28, 136-156. (doi:10.1086/399512)

543 Wilson EO. 1984 The relation between caste ratios and division of labor in the ant

544 genus Pheidole (Hymenoptera: Formicidae). Behav Ecol Sociobiol 16, 89-98.

545 (doi:10.1007/BF00293108) 
bioRxiv preprint doi: https://doi.org/10.1101/2020.11.16.385393; this version posted November 17, 2020. The copyright holder for this preprint (which was not certified by peer review) is the author/funder, who has granted bioRxiv a license to display the preprint in perpetuity. It is made available under aCC-BY-NC-ND 4.0 International license.

\section{Supporting Information}

548 Table SI1: Characteristics of each finite element mesh.

\begin{tabular}{|c|c|c|c|c|}
\hline & Specimen & Mesh volume $\left(\mathrm{mm}^{3}\right)$ & Element edge length (mm) & Number of elements \\
\hline \multirow{2}{*}{ 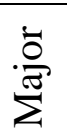 } & P. hetschkoi & 0.0741 & 0.023 & 449488 \\
\hline & $P$. cf. lucretii & 0.0183 & 0.023 & 278634 \\
\hline \multirow{2}{*}{$\stackrel{\overline{0}}{\stackrel{\Xi}{\Sigma}}$} & P. hetschkoi & 0.00469 & 0.0035 & 881691 \\
\hline & P. cf. lucretii & 0.00191 & 0.0035 & 392790 \\
\hline
\end{tabular}

